\title{
Arc-discharge ion sources for heavy ion fusion
}

\author{
André Anders and Joe W. Kwan \\ Lawrence Berkeley National Laboratory, University of California, \\ Berkeley, CA 94720, USA
}

\begin{abstract}
A miniature multiple beamlet approach to an injector system was recently proposed in order to reduce the size, cost, and power requirements of the injector. The beamlets of very high current density are needed to meet the brightness requirement. Besides vacuum arc ion sources, cold-cathode gas ion sources are candidates for this application. Vacuum-arc metal ion sources and vacuum-arc-like gas ion sources are discussed. Experiments are presented that focus on the short-pulse plasma composition and ion charge state distribution. $\mathrm{Mg}$ and $\mathrm{Sr}$ have been identified as the most promising metals leading to mono-species beams when $20 \mu \mathrm{s}$ arc pulses are used. It is shown that the efficient production of gas ions requires the presence of a magnetic field.
\end{abstract}


LBNL-44483

\section{Introduction}

Heavy ion driven inertial fusion (short: heavy ion fusion, HIF) is based on the compression and heating of fusionable material by powerful ions beams. Conceptually, an array of about 100 ion beams is used, with ion energies in the multi-GeV range and current of 0.5 A per beam. Two options of building injectors for HIF drivers are currently under consideration. The traditional way is to use low current density, large aperture, contact ionization sources. The major disadvantage of this approach is the very large size of the injector and matching section. The other option is to use high current density, multiple beamlet ion sources. From various scaling rules, it is found ${ }^{1}$ that the multiple beamlet approach is more attractive because the system can be smaller and at lower cost, although the requirements on the ion source are more demanding. In this paper, high current density ion sources are discussed that are based on arc plasma discharges and that may meet the requirements of HIF. The paper is organized as follows: we start by defining the requirements that ion sources must meet for the highcurrent-density option. Two types of arc ion sources are briefly reviewed: the vacuum arc (Mevva) ion source, and gas arc ion sources. Preliminary experimental results recently obtained at LBNL are summarized, followed by concluding remarks.

\section{Requirements}

The ion source for each of the about 100 beams must supply $~ 0.5 \mathrm{~A}$ of heavy ion beam current at a repetition rate of up to 10 p.p.s. The beam pulse length is about $20 \mu \mathrm{s}$ with a risetime preferably less than $1 \mu \mathrm{s}$. Typical ions of interest (based on target penetration range) are $\mathrm{Bi}^{+}, \mathrm{Pb}^{+}, \mathrm{Hg}^{+}, \mathrm{Cs}^{+}, \mathrm{Xe}^{+}$, and $\mathrm{Rb}^{+}$; higher charge states are also of 
interest in some driver designs. Some lighter ions such as $\mathrm{K}^{+}, \mathrm{Ar}^{+}$and $\mathrm{Ne}^{+}$can be useful because they provide an opportunity to do experiments at driver-scale ion velocities on medium length accelerators during the next development phase of HIF facilities.

In order to focus the ion beams onto a mm-size fusion target and also to allow for emittance growth during acceleration, the desired normalized emittance at the injector is generally $<1.0$ ðmm-mrad. Such low emittance can be achieved by using small aperture (i.e. high current density) ion sources. Previous considerations ${ }^{1}$ showed that the current density of each beamlet should reach $100 \mathrm{~mA} / \mathrm{cm}^{2}$.

Additionally to the above requirements, the HIF ion source

a must deliver pulses of desired ion species with negligible amounts of contaminating species (contamination includes "foreign" elements as well as unwanted charge states of the wanted element; ideally the element of choice has only one isotope),

must have good pulse-to-pulse reproducibility (1\% rms or better),

must produce a beam of stable current during each pulse (i.e. low noise, with $1 \% \mathrm{rms}$ or better),

must not operate at high gas pressures because charge exchange and other collisional processes degrade beam quality; the pressure at the extractor system should be less than $\sim 10^{-4}$ Torr (less than $\sim 10 \mathrm{mPa}$ ).

\section{Vacuum arc (Mevva) ion sources}

A vacuum arc ion source (also known as metal vapor vacuum arc (Mevva) ion source ${ }^{2,3}$ ) is an attractive option for HIF because it can produce ions of practically all conductive solids, it does not require any gas, its ion beams can have high current 
density, and multiply charged ions can be produced. Even in their early stage of development more than a decade ago, vacuum arc ion sources had been considered for HIF application ${ }^{4,5}$. Early results were mixed. Despite the above mentioned advantages, Mevva ions sources became known for high beam noise and poor pulse-to-pulse reproducibility. Attempts have been made to solve these problems with grid control of the plasma. Indeed, good results have been obtained this way by Humphries and

coworkers ${ }^{6,7}$ but the survival time of the very fine grids and meshes was unsatisfactory. More recently, Oks and coworkers at GSI combined stabilizing grids and fine meshes ${ }^{8,9}$, demonstrating a significant reduction of beam noise.

In a recent testing of the Mevva for HIF application by Liu et al. ${ }^{10}$, up to 24 $\mathrm{mA} / \mathrm{cm}^{2}$ of $\mathrm{Gd}$ ions were produced with about $80 \%$ of the ions in the $2+$ state and a pulse rise-time close to $1 \mu \mathrm{s}$. The results showed a significant improvement in the beam noise suppression and shot-to-shot reproducibility (3\% rms under optimum conditions). More recent results by Qi et al. ${ }^{11}$ confirmed that the Mevva ion source is indeed a good candidate that could meet the requirements of the high-current density HIF driver option. More advantages and issues of vacuum arc ion sources are presented and discussed in the experimental section below.

\section{Gas arc ion sources}

Arc sources based on high-current, cold-cathode operation can produce copious quantities of plasma, ions, and beam current in very short times. Risetimes are limited by the impedance of the discharge system, not by the ionization time. The very short 
transient time is an interesting peculiarity which is in contrast to other ion sources and therefore worth of some clarification.

Following the ionization theory of Donets ${ }^{12}$, the likelihood that an atom becomes ionized can be expressed in terms of the electron current density in the discharge, $j_{e}$, and the duration the atom is subject to the electron flux, $\tau$,

$$
P=C \frac{j_{e}}{e} \tau
$$

where $C$ is a value related to the ionization cross section at the energy of electrons. At electron energies of typically $10-50 \mathrm{eV}$, corresponding to the "tail" if the electrons are Maxwell-distributed with a few eV temperature, the value of $C$ is of order $10^{16} \mathrm{~cm}^{-2}$ for singly and doubly charged ions ${ }^{12}$. We consider two limiting cases. In gas discharge plasmas, a typical current density is $j_{e}=1 \mathrm{~A} / \mathrm{cm}^{2}$ or less. If we asked for the characteristic time an atom needs to become ionized with very high probability $(P \rightarrow 1)$, we obtain from (1) that $\tau \approx 1 \mathrm{~ms}$. In contrast, the current density at a spot of a coldcathode arc discharge, regardless of vacuum or low-pressure arc, is of order $j_{e}=10^{6} \mathrm{~A} / \mathrm{cm}^{2}$ or even higher, and the characteristic ionization time is therefore $\tau \approx 1 \mathrm{~ns}$ or less. Even with the uncertainty of the exact value of the current densities, this estimate shows clearly that the high current density at cathode spots is a necessary condition for fast risetime of plasma production.

The above estimate suggests that a Mevva-like ion source could operate as a fast responding gas ion source if gas ionization can be accomplished while metal ions are suppressed at the extractor system. It has been reported by several researchers ${ }^{13,14}$ that vacuum arc plasma and ion sources can produce large amounts of gaseous (as opposed to 
metal) ions when gas is added to the arc discharge region. This has been used for hybrid metal and gas ion implantation in materials ${ }^{15,16}$.

Cold-cathode arc discharges have been used as electron ${ }^{17-19}$ and hydrogen ion 20,21 sources. In all of these sources, a gas puff valve was used to temporarily obtain a high pressure in the discharge region. Beam pulses are usually of millisecond duration but pulses as short as $200 \mu$ s have been demonstrated. Although high current, high brightness beams have been obtained, the source operation cannot be directly used because gas puff valves are too slow for the $1 \mu$ s risetime, $20 \mu$ s duration pulses required for HIF.

For completeness of this overview one should mention that $20 \mu$ s pulses for HIF can be accomplished by keeping the plasma (ion reservoir) at steady-state conditions while pulsing the extractor system, or the supply of ions via a biasing technique ${ }^{22}$. Perkins et al. ${ }^{22}$ maintained the plasma using a thermionic (hot-filament) arc discharge. Risetimes close to $1 \mu \mathrm{s}$ have been achieved though for very low current density of 0.5 $\mathrm{mA} / \mathrm{cm}^{2}$

\section{Experimental results}

\subsection{Vacuum arc ion source}

The majority of results is presented in a companion paper ${ }^{11}$. Here we focus on measurements of ion beam composition and charge state distribution while our system is being upgraded for future current density and emittance measurements.

Ion charges state distributions (CSDs) are tabulated for almost all conducting elements ${ }^{3}$. However, these data are compiled for quasi-steady-state conditions which are 
established when the vacuum arc operates longer than about $100 \mu \mathrm{s}$. It is known that the ion charge states are higher at the beginning of each arc pulse ${ }^{23,24}$ and therefore the data compiled in ${ }^{3}$ cannot be used for $20 \mu$ s pulses as needed for HIF. Time-of-flight (TOF) charge-to-mass spectrometry ${ }^{24,25}$ was used to investigate short-pulse CSDs (Fig. 1). Unless stated otherwise, the following experimental conditions were used. The arc current was 300 A with a rise time of $1.0 \mu$ s $(10 \% \rightarrow 90 \%$ rise $), 16 \mu$ s flat portion, and a fall time of $5 \mu$ s $(90 \% \rightarrow 10 \%$ fall). The repetition rate was 2 p.p.s. The extractor voltage was $50 \mathrm{kV}$. Experiments were performed in high vacuum of about $0.13 \mathrm{mPa}\left(10^{-}\right.$ 6 Torr).

It was found that CSDs were shifted to higher charge states than steady-state values, and they showed a significant change during the $20 \mu$ s pulse. Figure 2 illustrates the situation for gold ions. Most of the early charge state enhancement is due to the power dissipation in the arc plasma which is higher in the transient initiation phase. Although gold has only one isotope, it obviously is not a good choice because the spectrum contains several charge states and is not constant.

In our search for mono-charge-state plasmas, many elements have been tested ${ }^{26}$. Only two elements have been identified as candidates for the production of ion beams with a single ion species $\mathrm{Sr}^{2+}$ and $\mathrm{Mg}^{2+}$.

Figure 3 shows the spectrum of strontium as an example of a mono-species vacuum-arc beam. While up to $15 \%$ is $\mathrm{Sr}^{3+}$ for the first few microseconds of each arc pulse, the CSD is dominated by charge state $2+$ for most of the pulse (charge state percent are expressed with respect to total particle current). Unfortunately, strontium 
oxidizes easily, and strontium oxide is toxic. The spectrum shows measurable amounts of hydrogen and oxygen.

Although lighter than the ideal ion species, $\mathrm{Mg}$ could be of use for intermediate driver experiments. At the beginning of each pulse, only $\mathrm{Mg}^{2+}$ is present but up to $25 \%$ of $\mathrm{Mg}^{1+}$ can be observed at the end of a $20 \mu$ s pulse.

Contamination with non-metallic species is significant for some of the elements. When the arc discharge starts, oxide layers and the water film are removed (eroded) from the cathode surface. The typically lighter elements arrive earlier at the ion extraction system than the heavier metallic species. As a result, the pulsed ion beam can be dominated by non-metallic species for the first few microseconds. Figure 4 shows an extreme case: the hafnium ion beam spectrum at $3 \mu$ s does not show any hafnium while hafnium is dominant at $17 \mu$ s after arc initiation.

A much larger number of mono-species vacuum arc plasmas can be obtained when the requirement of a $20 \mu$ s arc pulse is dropped by switching the beam pulse. In steady-state plasmas, some cathode elements have almost exclusively one charge state, e.g. 2+ (barium) and 1+ (bismuth at low arc current).

\subsection{Gas arc ion source}

As pointed out above, the high current density of cold-cathode arcs can provide rapid ionization. Mevva experiments with background gas have shown that gas ions can be produced. However, gas ion production without magnetic field is very inefficient, as illustrated in Figure 5. 
Interestingly, the absolute beam current seems to increase when adding a little background gas to the system: see top spectrum (high vacuum, $1 \times 10^{-6}$ Torr) and center spectrum (Argon, $2.5 \times 10^{-5}$ Torr). This effect can be attributed to the faster formation of secondary electrons that help to compensate the beam's space charge. The TOF gate is located about $0.75 \mathrm{~m}$ from the extractor system, thereby only a small part of the ion beam is utilized if space charge blow-up is severe. The formation of secondary electrons reduces blow-up and thus apparently higher currents are measured under conditions that allow fast formation of secondary electrons. In actual injector schemes, ions are postaccelerated immediately after extraction. Their density and space charge drop quickly and therefore beam blow-up as observed here should not occur.

The formation of gas ions is greatly enhanced if an axial magnetic field is added (Fig. 6). The ionization zone is extended from the vicinity of the cathode spots to a larger zone adjacent to the spots. The interaction time of metal ions and emitted electrons with gas atoms is much larger in the presence of a magnetic field, and additionally the power pumped into the discharge is enhanced (as one can see by the enhanced arc voltage ${ }^{27-30}$ ). To utilize this effect, our vacuum arc ion source was equipped with a magnetic field coil (0.1 mT/A) that could be powered independently from the arc current by using a second, thyristor-switched PFN. The arc pulse $(20 \mu \mathrm{s})$ was placed in the maximum of the field pulse $(250 \mu$ s pulse). The magnetic field can be considered constant (maximum $42 \mathrm{mT}$ ) for the duration of the arc. Each spectrum (such as in Fig. 6) was taken $10 \mu$ s after the first ions arrive at the TOF gate. We see that hydrogen is much more pronounced when a magnetic field is used ${ }^{31}$. Gas ions are efficiently ionized, and gas ions can dominate the 
beam at sufficiently high gas pressure and magnetic field. In the example of Figure 6, less than $3 \%$ are metal ions.

\section{Acknowledgments}

This work was supported by the U.S. Department of Energy, under Contract No. DE-AC03-76SF00098. 


\section{Figure Captions}

Figure 1 Ion source with time-of-flight (TOF) charge-to-mass spectrometer (not to scale; the distance from cathode to the extractor is $14.5 \mathrm{~cm}$, from extractor or TOF gate is $75 \mathrm{~cm}$, and from TOF gate to TOF detector is $103 \mathrm{~cm}$ ).

Figure 2 Development of the charge state distribution of gold during a $20 \mu \mathrm{s}$ arc pulse; raw time-of-flight data for $300 \mathrm{~A}$ arc, measured after $4 \mu \mathrm{s}$ (upper charge state spectrum) and $16 \mu$ s (lower spectrum) after arrival of ions at the TOF gate. The numbers $1-5$ refer to the ion charge state of gold ions. The amplitude is electrical current in arbitrary units.

Figure 3 TOF spectrum of strontium (10 $\mu$ s after ion arrival at the TOF gate) as an example of a mono-species vacuum-arc beam.

Figure 4 TOF spectrum of hafnium ion beam: at $3 \mu$ s after arrival of ions at the TOF gate (upper spectrum) the beam is dominated by hydrogen and hafnium is absent, while at $17 \mu \mathrm{s}$ hafnium is dominant.

Figure 5 Aluminum vacuum arc with argon gas injection near cathode: Gas ion production is inefficient without magnetic field. Each of the three spectra was measured at $10 \mu \mathrm{s}$ after the first ions arrived at the TOF gate. The amplitude scale is the same for each spectrum, and the numbers 1-3 refer to the aluminum ion charge state.

Figure 7 TOF spectra from vacuum-arc ions source operating with a magnetic field as shown in Figure 5; operation with an aluminum cathode. Top: vacuum operation $\left(10^{-6}\right.$ Torr), bottom: argon gas flow $\left(5 \times 10^{-5}\right.$ Torr). Note the strong presence of hydrogen contamination and very small amount of aluminum when gas is added. 
LBNL-44483

\section{References}

${ }^{1}$ J.W. Kwan, Rev. Sci. Instrum. 71, 807-809 (2000).

${ }^{2}$ I.G. Brown, "The Metal Vapor Vacuum Arc Ion Source," in The Physics and Technology of Ion Sources, edited by I.G. Brown (John Wiley \& Sons, New York, 1989), pp. 331-354.

${ }^{3}$ I. G. Brown, Rev. Sci. Instrum. 65, 3061-3081 (1994).

${ }^{4}$ S. Humphries Jr. and H. Rutkowski, J. Appl. Phys. 67, 3223-3232 (1990).

${ }^{5}$ S. Humphries Jr. and C. Burkhart, Particle Accelerators 20, 211-228 (1987).

${ }^{6}$ S. Humphries Jr., C. Burkhart, S. Coffey, G. Cooper et al., J. Appl. Phys. 59, 1790-1798 (1986).

${ }^{7}$ S. Humphries Jr., C. Burkhart, and L.K. Len, "Ion Sources for Pulsed High-Brightness Beams," in The Physics and Technology of Ion Sources, edited by I.G. Brown (John Wiley \& Sons, New York, 1989), pp. 397-419.

${ }^{8}$ E. Oks, P. Spädtke, H. Emig, and B.H. Wolf, Rev. Sci. Instrum. 65, 3109-3112 (1994).

${ }^{9}$ H. Reich, P. Spädtke, and E.M. Oks, Rev. Sci. Instrum. 71, 707-709 (2000).

${ }^{10}$ F. Liu, N. Qi, S. Gensler, R.R. Prasad, and M. Krishnan, Rev. Sci. Instrum. 69, 819-821 (1998).

${ }^{11}$ N. Qi, J. Schein, R.R. Prasad, M. Krishnan, A. Anders, J. Kwan, and I. Brown, to be published in Nucl. Instrum. Meth. Phys. Res. A, as part of Proceedings of the Int. Workshop of Heavy Ion Fusion, San Diego, March 13-17, 2000 (2000).

${ }^{12}$ E.D. Donets, "Electron Beam Ion Sources," in The Physics and Technology of Ion Sources, edited by I.G. Brown (John Wiley, New York, 1989), pp. 245-279. 
LBNL-44483

${ }^{13}$ C. A. Davies, M. K. Puchert, D. R. McKenzie, and B. W. James, "The filtered cathodic arc as a source of gas ions," Proc. XVth Int. Symp. on Discharges and Electrical Insulation in Vacuum, Darmstadt, Germany, 1992, 690-693.

${ }^{14}$ P. Spädtke, H. Emig, B.H. Wolf, and E. Oks, Rev. Sci. Instrum. 65, 3113-3118 (1994).

${ }^{15}$ E. Oks, G. Yushkov, P. Schanin, and A. Nikolaev, Rev. Sci. Instrum. 67, 1213-1215 (1996).

${ }^{16}$ I.G. Brown, F. Liu, O.R. Monteiro, K.M. Yu et al., Surf. \& Coat. Technol. 103-104, 293-298 (1998).

${ }^{17}$ M. Hamagaki, T. Hara, Y. Sadamoto, and T. Ohgo, Rev. Sci. Instrum. 66, 3469-3471 (1995).

${ }^{18}$ G. Fiksel, A.F. Almagri, D. Craig, M. Lida, S.C. Prager, and J.S. Sarff, Plasma Sources Sci. Technol. 5, 78-85 (1996).

${ }^{19}$ D.J. Den Hartog, D.J. Craig, G. Fiksel, and J.S. Sarff, Plasma Sources Sci. Technol. 6, 492-498 (1997).

${ }^{20}$ Yu.I. Belchenko, V.I. Davydenko, G.E. Derevyankin, G.I. Dimov et al., Rev. Sci. Instrum. 61, 378-384 (1990).

${ }^{21}$ V.I. Davydenko, Nucl. Instrum. Meth. Phys. Res. A 427, 230-234 (1999).

${ }^{22}$ L.T. Perkins, J.W. Kwan, K.N. Leung, M. Rickard, and M.D. Williams, Rev. Sci. Instrum. 69, 1060-1062 (1998).

${ }^{23}$ J. E. Galvin, I. G. Brown, and R. A. MacGill, Rev. Sci. Instrum. 61, 583-585 (1990).

${ }^{24}$ A. Anders, IEEE Trans. Plasma Sci. 26, 118-119 (1998).

${ }^{25}$ I. G. Brown, J. E. Galvin, R. A. MacGill, and R. T. Wright, Rev. Sci. Instrum 58, 15891592 (1987). 
${ }^{26} \mathrm{~A}$. Anders, "A periodic table of ion charge state distributions observed in the transition region between vacuum sparks and vacuum arcs," Proc. Int. Symposium on Discharges and Electrical Insulation in Vacuum, Xi;an, P.R. China, 2000.

${ }^{27}$ E. Oks, I. G. Brown, M. R. Dickinson, R. A. MacGill, P. Spädtke, H. Emig, and B. H. Wolf, Appl. Phys. Lett. 67, 200-202 (1995).

${ }^{28}$ E. Oks, A. Anders, I. Brown, M. Dickinson, and R. MacGill, "Influence of a strong pulsed magnetic field on the charge state distribution of ions in a vacuum arc plasma," Proc. XVIIth Int. Symp. Discharges and Electrical Insulation in Vacuum, Berkeley, 1996, 137-140.

${ }^{29}$ F.J. Paoloni and I. G. Brown, Rev Sci. Instrum. 66, 3855-3858 (1995).

${ }^{30}$ A. Anders, G. Yushkov, E. Oks, A. Nikolaev, and I. Brown, Rev. Sci. Instrum. 69, 1332-1335 (1998).

${ }^{31}$ J. M. Schneider, A. Anders, I.G. Brown, B. Hjörvarsson, and L. Hultman, Appl. Phys. Lett. 75, 612-614 (1999). 